\title{
Iterated Extensions and Uniserial Length Categories
}

\section{Eivind Eriksen ${ }^{1}$}

Received: 30 October 2018 / Accepted: 8 January 2020 / Published online: 19 March 2020

(C) The Author(s) 2020

\begin{abstract}
In this paper, we study length categories using iterated extensions. We fix a field $k$, and for any family $S$ of orthogonal $k$-rational points in an Abelian $k$-category $\mathcal{A}$, we consider the category $\operatorname{Ext}(\mathrm{S})$ of iterated extensions of $S$ in $\mathcal{A}$, equipped with the natural forgetful functor $\operatorname{Ext}(\mathrm{S}) \rightarrow \mathcal{A}(\mathrm{S})$ into the length category $\mathcal{A}(\mathrm{S})$. There is a necessary and sufficient condition for a length category to be uniserial, due to Gabriel, expressed in terms of the Gabriel quiver (or Ext-quiver) of the length category. Using Gabriel's criterion, we give a complete classification of the indecomposable objects in $\mathcal{A}(\mathrm{S})$ when it is a uniserial length category. In particular, we prove that there is an obstruction for a path in the Gabriel quiver to give rise to an indecomposable object. The obstruction vanishes in the hereditary case, and can in general be expressed using matric Massey products. We discuss the close connection between this obstruction, and the noncommutative deformations of the family $\mathrm{S}$ in $\mathcal{A}$. As an application, we classify all graded holonomic $D$-modules on a monomial curve over the complex numbers, obtaining the most explicit results over the affine line, when $D$ is the first Weyl algebra. We also give a non-hereditary example, where we compute the obstructions and show that they do not vanish.
\end{abstract}

Keywords Finite length categories · Uniserial categories · Iterated extensions · Noncommutative deformations

Mathematics Subject Classification (2010) Primary 18E10; Secondary 16G99 · 14F10

\section{Introduction}

Let $S=\left\{S_{\alpha}: \alpha \in I\right\}$ be a family of non-zero, pairwise non-isomorphic objects in an Abelian $k$-category $\mathcal{A}$, where $k$ is a field. We consider the minimal full subcategory $\mathcal{A}(\mathrm{S}) \subseteq \mathcal{A}$ that contains $\mathrm{S}$ and is closed under extensions. The family $\mathrm{S}$ is called a family of orthogonal points if $\operatorname{End}\left(S_{\alpha}\right)$ is a division algebra and $\operatorname{Hom}\left(S_{\alpha}, S_{\beta}\right)=0$ for all $\alpha, \beta \in I$

Presented by: Michel Brion

Eivind Eriksen

eivind.eriksen@bi.no

1 Department of Economics, BI Norwegian Business School, Oslo, Norway 
with $\alpha \neq \beta$. In this case, $\mathcal{A}(\mathrm{S}) \subseteq \mathcal{A}$ is a length category with $\mathrm{S}$ as its simple objects. When $\operatorname{End}\left(S_{\alpha}\right)=k$, we call $S_{\alpha}$ a $k$-rational point.

An important special case is when $\mathcal{A}=\operatorname{Mod}_{A}$ is the category of modules over an associative $k$-algebra $A$, and $\mathrm{S}$ is a subset of the simple $A$-modules. If $\mathrm{S}$ is the family of all simple modules, then $\mathcal{A}(\mathrm{S})$ is the category of all modules of finite length. There are also many other interesting applications, for example when $\mathcal{A}$ is the category of graded modules over a graded $k$-algebra, or the category of coherent sheaves over a $k$-scheme. Note that any length category is exact equivalent to an exact subcategory of a module category. Nevertheless, it is often better to work directly in the Abelian category of interest than to use an embedding into a module category.

We shall use the category $\operatorname{Ext}(\mathrm{S})$ of iterated extensions of $\mathrm{S}$ to study the length category $\mathcal{A}(\mathrm{S})$ when $\mathrm{S}$ is a family of orthogonal $k$-rational points. An iterated extension of $\mathrm{S}$ is a couple $(X, C)$, where $X$ is an object in $\mathcal{A}$ and $C$ is a cofiltration

$$
X=C_{n} \stackrel{f_{n}}{\rightarrow} C_{n-1} \rightarrow \cdots \rightarrow C_{2} \stackrel{f_{2}}{\rightarrow} C_{1} \stackrel{f_{1}}{\rightarrow} C_{0}=0
$$

where $f_{i}: C_{i} \rightarrow C_{i-1}$ is surjective and $K_{i}=\operatorname{ker}\left(f_{i}\right) \cong S_{\alpha_{i}}$ with $\alpha_{i} \in I$ for $1 \leq i \leq n$. The assignment $(X, C) \mapsto X$ defines a forgetful functor $\operatorname{Ext}(\mathrm{S}) \rightarrow \mathcal{A}(\mathrm{S})$.

The category $\operatorname{Ext}(S)$ of iterated extensions has some interesting invariants, in addition to the length $n$, the simple factors $\left\{K_{1}, \ldots, K_{r}\right\}$ with $r \leq n$, and their multiplicities. The order vector $\boldsymbol{\alpha}=\left(\alpha_{1}, \ldots, \alpha_{n}\right) \in I^{n}$ is an invariant of $\operatorname{Ext}(\mathrm{S})$. Moreover, when $(X, C)$ is an iterated extension with order vector $\boldsymbol{\alpha}$, there are induced commutative diagram of short exact sequences

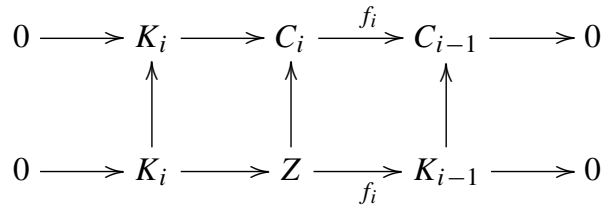

We call the extensions $\tau_{i} \in \operatorname{Ext}_{\mathcal{A}}^{1}\left(K_{i-1}, K_{i}\right)$ for $2 \leq i \leq n$ the simple extensions of $(X, C)$. These simple extensions are also invariants of $\operatorname{Ext}(\mathrm{S})$.

We say that $\mathcal{A}(\mathrm{S})$ is a uniserial length category if any indecomposable object in $\mathcal{A}(\mathrm{S})$ has a unique composition series. When $S$ is a family of $k$-rational orthogonal points, then $\mathcal{A}(\mathrm{S})$ is a uniserial length category if and only if $S$ satisfies the following criterion, due to Gabriel:

$$
\sum_{\beta \in I} \operatorname{dim}_{k} \operatorname{Ext}_{\mathcal{A}}^{1}\left(S_{\alpha}, S_{\beta}\right) \leq 1 \text { and } \sum_{\beta \in I} \operatorname{dim}_{k} \operatorname{Ext}_{\mathcal{A}}^{1}\left(S_{\beta}, S_{\alpha}\right) \leq 1 \quad \text { for all } \alpha \in \mathrm{S}
$$

The Gabriel quiver of $\mathcal{A}(\mathrm{S})$ has nodes $\mathrm{S}=\left\{S_{\alpha}: \alpha \in I\right\}$, and $\operatorname{dim}_{k} \operatorname{Ext}_{\mathcal{A}}^{1}\left(S_{\alpha}, S_{\beta}\right)$ edges from node $\alpha$ to node $\beta$. Gabriel's criterion for $\mathcal{A}(\mathrm{S})$ to be uniserial is a condition on the Gabriel quiver, and we use it to classify and explicitly construct all indecomposable objects in $\mathcal{A}(\mathrm{S})$ when the length category is uniserial.

Let $n \geq 2$, and assume that simple objects $K_{1}, \ldots, K_{n}$ in S and extensions $\tau_{2}, \ldots, \tau_{n}$ in $\mathcal{A}$ are given, such that $\tau_{i} \in \operatorname{Ext}_{\mathcal{A}}^{1}\left(K_{i-1}, K_{i}\right)$ for $2 \leq i \leq n$. If $n=2$, then it is clear that any extension of $K_{1}$ by $K_{2}$ is an iterated extension in $\operatorname{Ext}(\mathrm{S})$ with simple factors $K_{1}, K_{2}$ and simple extension $\tau_{2}$. For $n \geq 3$, we give a necessary and sufficient condition for the existence of an iterated extension in $\operatorname{Ext}(\mathrm{S})$ with simple factors $K_{1}, \ldots, K_{n}$ and simple extensions $\tau_{2}, \ldots, \tau_{n}$. The condition is given in terms of matrix Massey products in the sense of May, and we show that it can be interpreted as obstructions for lifting infinitesimal noncommutative deformation. In order to do this, we describe the close link between iterated extensions and noncommutative deformations, following Laudal [10]. 
Theorem Let $\mathrm{S}=\left\{S_{\alpha}: \alpha \in I\right\}$ be a family of orthogonal $k$-rational points in an Abelian $k$-category $\mathcal{A}$. If $\mathcal{A}(\mathrm{S})$ is a uniserial length category, then the indecomposable objects in $\mathcal{A}(\mathrm{S})$ of length $n$ are given by

$$
\left\{X(\boldsymbol{\alpha}): \boldsymbol{\alpha} \in I^{n} \text { is admissible }\right\}
$$

up to isomorphism in $\mathcal{A}(\mathrm{S})$. Moreover, an order vector $\alpha \in I^{n}$ is admissible if and only if it satisfies the following conditions:

1. The order vector $\alpha$ corresponds to a path of length $n-1$ in the Gabriel quiver $\Lambda$.

2. When $n \geq 3$, the matric Massey product $\left\langle\tau_{2}, \tau_{3}, \ldots, \tau_{n}\right\rangle \subseteq \operatorname{Ext}_{\mathcal{A}}^{2}\left(S_{\alpha_{1}}, S_{\alpha_{n}}\right)$ is defined and contains zero for all non-split extensions $\tau_{i} \in \operatorname{Ext}_{\mathcal{A}}^{1}\left(S_{\alpha_{i-1}}, S_{\alpha_{i}}\right)$.

When $\mathcal{A}(\mathrm{S})$ is hereditary, the obstructions vanish, and any path of length $n-1$ in the Gabriel quiver of $\mathcal{A}(\mathrm{S})$ gives rise to an indecomposable object of length $n$ in $\mathcal{A}(\mathrm{S})$. Consequently, there is a bijective correspondence between indecomposable objects of length $n$ in $\mathcal{A}(\mathrm{S})$ and paths of length $n-1$ in the Gabriel quiver of $\mathcal{A}(\mathrm{S})$. This result is well-known; see for instance Chen and Krause [3].

As an application, we show that the category $\mathbf{g r H o l}_{D}$ of graded holonomic $D$-modules is uniserial when $D=\operatorname{Diff}(A)$ is the ring of differential operators on a monomial curve $A$ defined over the field $k=\mathbb{C}$ of complex numbers. Moreover, we classify all indecomposable objects in $\mathbf{g r H o l}_{D}$. We build upon the results in Eriksen [6], where we studied this category. We obtain the most explicit result in the case when $A=k[t]$ and $D=A_{1}(k)$ is the first Weyl algebra. The classification is similar in the other cases, since all rings of differential operators on monomial curves are Morita equivalent.

Theorem Let $D=A_{1}(k)$ be the first Weyl algebra. Then the category $\mathbf{g r H o l}_{D}$ of graded holonomic D-modules is uniserial, and the indecomposable D-modules in $\mathbf{g r H o l}_{D}$ are, up to graded isomorphisms and twists, given by

$$
M(\alpha, n)=D / D(E-\alpha)^{n}, \quad M(\beta, n)=D / D \mathbf{w}(\beta, n)
$$

where $n \geq 1, \alpha \in J^{*}=\{\alpha \in k: 0 \leq \operatorname{Re}(\alpha)<1, \alpha \neq 0\}, \beta \in\{0, \infty\}$, and where $E=t \partial$ is the Euler derivation and $\mathbf{w}(\beta, n)$ is the alternating word on $n$ letters in $t$ and $\partial$, ending with $\partial$ if $\beta=0$, and in $t$ if $\beta=\infty$.

This result resembles Boutet de Monvel's classical result, giving all regular holonomic $\mathcal{D}$-modules over the ring $\mathcal{D}$ of differential operators on the ring $\mathcal{O}$ of germs of holomorphic function on $X=\mathbb{C}$ around 0 .

We also include an example of a non-hereditary uniserial length category, and compute the obstructions. The simplest example is $A=k[x] /\left(x^{2}\right)$ with the family $S=\{S\}$ consisting of the unique simple left $A$-modules, given by $S=A /(x)$. We show that in this case, the obstructions do not vanish, and there are no indecomposable modules of length $n \geq 3$.

\section{Iterated Extensions and Length Categories}

Let $k$ be a field, let $\mathcal{A}$ be an Abelian $k$-category, and let $S=\left\{S_{\alpha}: \alpha \in I\right\}$ be a fixed family of non-zero, pairwise non-isomorphic objects in $\mathcal{A}$. In this section, we define the category $\operatorname{Ext}(\mathrm{S})$ of iterated extensions of the family $\mathrm{S}$, equipped with a forgetful functor $\operatorname{Ext}(\mathrm{S}) \rightarrow \mathcal{A}(\mathrm{S})$ into the minimal full subcategory $\mathcal{A}(\mathrm{S}) \subseteq \mathcal{A}$ that contains $\mathrm{S}$ and is closed under extensions, and study its properties. 
An object of $\operatorname{Ext}(S)$ is a couple $(X, C)$, where $X$ is an object of the category $\mathcal{A}$ and $C$ is a cofiltration of $X$ in $\mathcal{A}$ of the form

$$
X=C_{n} \stackrel{f_{n}}{\rightarrow} C_{n-1} \rightarrow \cdots \rightarrow C_{2} \stackrel{f_{2}}{\rightarrow} C_{1} \stackrel{f_{1}}{\rightarrow} C_{0}=0
$$

where $f_{i}: C_{i} \rightarrow C_{i-1}$ is surjective and $K_{i}=\operatorname{ker}\left(f_{i}\right) \cong S_{\alpha_{i}}$ with $\alpha_{i} \in I$ for $1 \leq i \leq n$. The integer $n \geq 0$ is called the length, the objects $K_{1}, \ldots, K_{n}$ are called the factors, and the vector $\boldsymbol{\alpha}=\left(\alpha_{1}, \ldots, \alpha_{n}\right)$ is called the order vector of the iterated extension $(X, C)$.

Let $(X, C)$ and $\left(X^{\prime}, C^{\prime}\right)$ be a pair of objects in $\operatorname{Ext}(\mathrm{S})$ of lengths $n, n^{\prime} \geq 0$. A morphism $\phi:(X, C) \rightarrow\left(X^{\prime}, C^{\prime}\right)$ in $\operatorname{Ext}(\mathrm{S})$ is a collection $\left\{\phi_{i}: 0 \leq i \leq N\right\}$ of morphisms $\phi_{i}: C_{i} \rightarrow$ $C_{i}^{\prime}$ in $\mathcal{A}$ such that $\phi_{i-1} f_{i}=f_{i}^{\prime} \phi_{i}$ for $1 \leq i \leq N$, where $N=\max \left\{n, n^{\prime}\right\}$. By convention, $C_{i}=X$ for all $i>n$ and $C_{i}^{\prime}=X^{\prime}$ for all $i>n^{\prime}$.

The category $\operatorname{Ext}(S)$ has a dual category defined by filtrations. An object of this category is a couple $(X, F)$, where $X$ is an object of $\mathcal{A}$ and $F$ is a filtration of $X$ in $\mathcal{A}$ of the form

$$
0=F_{n} \subseteq F_{n-1} \subseteq \cdots \subseteq F_{0}=X
$$

such that $K_{i}=F_{i-1} / F_{i} \cong S_{\alpha_{i}}$ with $\alpha_{i} \in I$ for $1 \leq i \leq n$. Given an object $(X, F)$ in the dual category, the corresponding object in $\operatorname{Ext}(S)$ is $(X, C)$, where the cofiltration $C$ is defined by $C_{i}=X / F_{i}$ for $0 \leq i \leq n$, with the natural surjections $f_{i}: C_{i} \rightarrow C_{i-1}$. Conversely, if an object $(X, C)$ in $\operatorname{Ext}(S)$ is given, then the corresponding filtration of $X$ is given by $F_{i}=\operatorname{ker}\left(X \rightarrow C_{i}\right)$ for $0 \leq i \leq n$, where $X \rightarrow C_{i}$ is the composition $f_{i+1} \circ \cdots \circ f_{n}: C_{n} \rightarrow C_{i}$. It is clear from the construction that the dual objects $(X, C)$ and $(X, F)$ have the same length, the same factors, and the same order vector.

As the name suggests, the category $\operatorname{Ext}(S)$ can be characterized in terms of extensions. In fact, for any object $(X, C)$ in $\operatorname{Ext}(\mathrm{S})$ of length $n$ and for any integer $i$ with $2 \leq i \leq n$, the cofiltration $C$ induces a commutative diagram

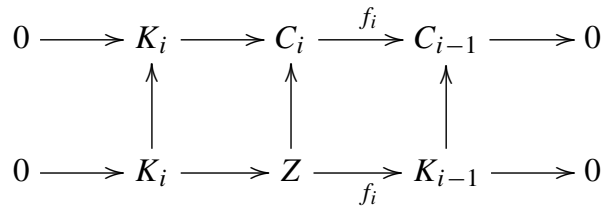

in $\mathcal{A}$, where the rows are exact and $Z=f_{i}^{-1}\left(K_{i-1}\right)$. We define $\xi_{i} \in \operatorname{Ext}_{\mathcal{A}}^{1}\left(C_{i-1}, K_{i}\right)$ and $\tau_{i} \in \operatorname{Ext}_{\mathcal{A}}^{1}\left(K_{i-1}, K_{i}\right)$ to be the extensions corresponding to the upper and lower row, and call $\tau_{2}, \tau_{3}, \ldots, \tau_{n}$ the simple extensions of $(X, C)$. By construction, $\xi_{i} \mapsto \tau_{i}$ under the map $\operatorname{Ext}_{\mathcal{A}}^{1}\left(C_{i-1}, K_{i}\right) \rightarrow \operatorname{Ext}_{\mathcal{A}}^{1}\left(K_{i-1}, K_{i}\right)$ induced by the inclusion $K_{i-1} \subseteq C_{i-1}$. In particular, $C_{2}$ is an extension of $C_{1}=K_{1}$ by $K_{2}, C_{3}$ is an extension of $C_{2}$ by $K_{3}$, and in general, $C_{i+1}$ is an extension of $C_{i}$ by $K_{i+1}$ for $1 \leq i \leq n-1$. It follows that $X=C_{n}$ is obtained from the factors $\left\{K_{1}, \ldots, K_{n}\right\} \subseteq \mathrm{S}$ by an iterated use of extensions, and this justifies the name iterated extensions.

Let us consider the natural forgetful functor $\operatorname{Ext}(\mathrm{S}) \rightarrow \mathcal{A}$ given by $(X, C) \mapsto X$, and the full subcategory $\mathcal{A}(\mathrm{S}) \subseteq \mathcal{A}$ defined in the following way: An object $X$ in $\mathcal{A}$ belongs to $\mathcal{A}(\mathrm{S})$ if there exists a cofiltration $C$ of $X$ such that $(X, C)$ is an object of $\operatorname{Ext}(\mathrm{S})$. The following lemma proves that $\mathcal{A}(\mathrm{S}) \subseteq \mathcal{A}$ is the minimal full subcategory that contains $\mathrm{S}$ and is closed under extensions:

Lemma 1 Let $\left(X^{\prime}, C^{\prime}\right),\left(X^{\prime \prime}, C^{\prime \prime}\right)$ be iterated extensions of the family S. If $X$ is an extension of $X^{\prime}$ by $X^{\prime \prime}$ in $\mathcal{A}$, then there is a cofiltration $C$ of $X$ such that $(X, C)$ is an iterated extension of the family $S$. In particular, the full subcategory $\mathcal{A}(\mathrm{S}) \subseteq \mathcal{A}$ is closed under extensions. 
Proof Let us assume that $\left(X^{\prime}, C^{\prime}\right)$ and $\left(X^{\prime \prime}, C^{\prime \prime}\right)$ are iterated extensions of the family $\mathrm{S}$ of lengths $n^{\prime}, n^{\prime \prime}$. Since $X$ is an extension of $X^{\prime \prime}$ by $X^{\prime}$, we can construct a cofiltration of $X$ of length $n=n^{\prime}+n^{\prime \prime}$ in the following way: Let $f: X^{\prime} \rightarrow X$ and $g: X \rightarrow X^{\prime \prime}$ be the maps given by the extension $0 \rightarrow X^{\prime} \rightarrow X \rightarrow X^{\prime \prime} \rightarrow 0$, let $F^{\prime}$ be the filtration of $X^{\prime}$ dual to the cofiltration $C^{\prime}$, and let $F^{\prime \prime}$ be the filtration of $X^{\prime \prime}$ dual to the cofiltration $C^{\prime \prime}$. We define $F_{i}=g^{-1}\left(F_{i}^{\prime \prime}\right)$ for $0 \leq i \leq n^{\prime \prime}$, and $F_{i}=f\left(F_{i-n^{\prime \prime}}^{\prime}\right)$ for $n^{\prime \prime} \leq i \leq n$. Then $F$ is a filtration of $X$, and we have that $F_{i-1} / F_{i} \cong \operatorname{ker}\left(X \rightarrow C_{i-1}^{\prime \prime}\right) / \operatorname{ker}\left(X \rightarrow C_{i}^{\prime \prime}\right) \cong K_{i}^{\prime \prime}$ for $0 \leq i \leq n^{\prime \prime}$, and also that $F_{i-1} / F_{i} \cong K_{i-n^{\prime \prime}}^{\prime}$ for $n^{\prime \prime} \leq i \leq n$. Let $C$ be the cofiltration of $X$ dual to the filtration $F$. Then it follows by construction that $(X, C)$ is an iterated extension of the family $S$ of length $n$.

We recall that $\mathcal{A}(\mathrm{S}) \subseteq \mathcal{A}$ is called an exact Abelian subcategory if the inclusion functor $\mathcal{A}(\mathrm{S}) \rightarrow \mathcal{A}$ is an exact functor. It is well-known that this is the case if and only if $\mathcal{A}(\mathrm{S})$ is closed in $\mathcal{A}$ under kernels, cokernels and finite direct sums. It is clear that $\mathcal{A}(\mathrm{S})$ is closed under finite direct sums since it closed under extensions. But in general, it is not closed under kernels and cokernels.

Proposition 2 The full subcategory $\mathcal{A}(\mathrm{S}) \subseteq \mathcal{A}$ is an exact Abelian subcategory if and only if the following conditions hold:

1. $\operatorname{End}_{\mathcal{A}}\left(S_{\alpha}\right)$ is a division algebra for all $\alpha \in I$

2. $\operatorname{Mor}_{\mathcal{A}}\left(S_{\alpha}, S_{\beta}\right)=0$ for all $\alpha, \beta \in I$ with $\alpha \neq \beta$

If this is the case, then $\mathrm{S}$ is the set of simple objects in $\mathcal{A}(\mathrm{S})$, up to isomorphism.

Proof This follows from Theorem 1.2 in Ringel [13], and the preceding comments.

We use the notation from Ringel [13], and say that an object $X$ in $\mathcal{A}$ is a point if End $_{\mathcal{A}}(X)$ is a division ring, and that two points $X, Y$ in $\mathcal{A}$ are orthogonal if $\operatorname{Mor}_{\mathcal{A}}(X, Y)=$ $\operatorname{Mor}_{\mathcal{A}}(Y, X)=0$. We call $X$ a $k$-rational point if $\operatorname{End}_{\mathcal{A}}(X)=k$.

A length category is an Abelian category such that any of its objects has finite length, and such that the isomorphism classes of objects form a set. If $\mathrm{S}$ is a family of orthogonal points in an Abelian $k$-category $\mathcal{A}$, then it follows from Proposition 2 that $\mathcal{A}(\mathrm{S})$ is a length category, with $S$ as its simple objects. In fact, any length category which is an Abelian $k$-category is of this type.

\section{Obstructions for Existence of Iterated Extensions}

In this section, we assume that $\mathcal{A}$ is the category $\operatorname{Mod}_{A}$ of right modules over an associative $k$-algebra $A$, and let $\mathrm{S}=\left\{S_{\alpha}: \alpha \in I\right\}$ be a family of orthogonal points in $\operatorname{Mod}_{A}$. Let $n \geq 2$ be an integer, and fix simple $A$-modules $K_{1}, \ldots, K_{n}$ in $\mathrm{S}$ and extensions $\tau_{i} \in$ $\operatorname{Ext}_{A}^{1}\left(K_{i-1}, K_{i}\right)$ for $2 \leq i \leq n$. If $n=2$, then any extension of $K_{1}$ by $K_{2}$ is an iterated extension in $\operatorname{Ext}(\mathrm{S})$ with $K_{1}, K_{2}$ as its simple factors and $\tau_{2}$ as its simple extension. For $n \geq$ 3 , there are obstructions, and we give a necessary and sufficient condition for the existence of an iterated extension in $\operatorname{Ext}(\mathrm{S})$ with $K_{1}, \ldots, K_{n}$ as its simple factors and $\tau_{2}, \ldots, \tau_{n}$ as its simple extensions. These obstructions are expressed in terms of matric Massey products; see May [11]. 
We write $K=\oplus K_{i}$, and consider the Hochschild complex $\operatorname{HC}^{\bullet}\left(A, \operatorname{End}_{k}(K)\right)$ of $A$ with values in $\operatorname{End}_{k}(K)$ as a DGA (differential graded algebra) over $k^{r}$. It has a decomposition

$$
\operatorname{HC}^{n}\left(A, \operatorname{End}_{k}(K)\right)=\underset{i, j}{\oplus} \operatorname{HC}^{n}\left(A, \operatorname{Hom}_{k}\left(K_{i}, K_{j}\right)\right)
$$

A 1-cochain in this DGA is a $k$-linear map $\alpha: A \rightarrow \operatorname{End}_{k}(K)$, and it is a 1-cocycle if and only if it is a derivation. Multiplication of the 1-cochains $\alpha, \beta$ in the DGA is defined by the composition $\alpha \cdot \beta=\{(a, b) \mapsto \beta(b) \circ \alpha(a)\}$. It is well-known that its cohomology is given by

$$
\operatorname{HH}^{n}\left(A, \operatorname{End}_{k}(K)\right)=\bigoplus_{i, j} \operatorname{HH}^{n}\left(A, \operatorname{Hom}_{k}\left(K_{i}, K_{j}\right)\right) \cong \underset{i, j}{\oplus} \operatorname{Ext}_{A}^{n}\left(K_{i}, K_{j}\right)
$$

In particular, $\operatorname{Ext}_{A}^{1}\left(K_{i}, K_{j}\right) \cong \operatorname{Der}_{k}\left(A, \operatorname{Hom}_{k}\left(K_{i}, K_{j}\right)\right) / \operatorname{IDer}_{k}\left(A, \operatorname{Hom}_{k}\left(K_{i}, K_{j}\right)\right)$, where $\operatorname{IDer}_{k}(-,-)$ denotes the inner derivations.

We choose a derivation $\alpha_{i-1, i}: A \rightarrow \operatorname{Hom}_{k}\left(K_{i-1}, K_{i}\right)$ that represents $\tau_{i}$ in Hochschild cohomology for $2 \leq i \leq n$. The cup product $\tau_{2} \cup \tau_{3}=\left\langle\tau_{2}, \tau_{3}\right\rangle$ is the cohomology class of $\alpha_{12} \cdot \alpha_{23}$. It is also called a second order matric Massey product. If the cup-products $\left\langle\tau_{2}, \tau_{3}\right\rangle=\left\langle\tau_{3}, \tau_{4}\right\rangle=0$, then there are 1-cochains $\alpha_{13}$ and $\alpha_{24}$ such that

$$
d\left(\alpha_{13}\right)=\alpha_{12} \cdot \alpha_{23} \quad \text { and } \quad d\left(\alpha_{24}\right)=\alpha_{23} \cdot \alpha_{34}
$$

In that case, $\underline{\alpha}=\left\{\alpha_{12}, \alpha_{23}, \alpha_{34}, \alpha_{13}, \alpha_{24}\right\}$ is called a defining system for the third order matric Massey product $\left\langle\tau_{2}, \tau_{3}, \tau_{4}\right\rangle$, and the cohomology class of

$$
\tilde{\alpha}_{14}=\alpha_{13} \cdot \alpha_{34}+\alpha_{12} \cdot \alpha_{24}
$$

is the corresponding value of $\left\langle\tau_{2}, \tau_{3}, \tau_{4}\right\rangle$. Notice that this cohomology class may depend on the defining system. Higher order matric Massey products are defined similarly:

Definition A defining system for the matric Massey product $\left\langle\tau_{2}, \tau_{3}, \ldots, \tau_{n}\right\rangle$ is a family

$$
\underline{\alpha}=\left\{\alpha_{i j}: 1 \leq i<j \leq n,(i, j) \neq(1, n)\right\}
$$

of 1-cochains $\alpha_{i j}: A \rightarrow \operatorname{Hom}_{k}\left(K_{i}, K_{j}\right)$ such that $\alpha_{i-1, i}$ is a 1-cocycle that represents $\tau_{i}$ for $2 \leq i \leq n$, and such that

$$
d\left(\alpha_{i j}\right)=\widetilde{\alpha}_{i j}, \quad \text { with } \tilde{\alpha}_{i j}=\sum_{l=i+1}^{j-1} \alpha_{i l} \cdot \alpha_{l j}
$$

when $j-i>1$. The matric Massey product $\left\langle\tau_{2}, \tau_{3}, \ldots, \tau_{n}\right\rangle$ is defined if it has a defining system. In that case, $\left\langle\tau_{2}, \tau_{3}, \ldots, \tau_{n}\right\rangle$ is the collection of cohomology classes represented by

$$
\widetilde{\alpha}_{1 n}=\sum_{l=2}^{n-1} \alpha_{1, l} \cdot \alpha_{l, n}
$$

for some defining system $\underline{\alpha}$.

Let $E_{2}$ be a right $A$-module that is an extension of $K_{1}$ by $K_{2}$, such that there is a short exact sequence $0 \rightarrow K_{2} \rightarrow E_{2} \rightarrow K_{1} \rightarrow 0$. Then it is well-known that $E_{2} \cong K_{2} \oplus K_{1}$ considered as a vector space over $k$, and that the right action of $A$ is given by

$$
\left(m_{2}, m_{1}\right) \cdot a=\left(m_{2} \cdot a+\psi_{a}^{12}\left(m_{1}\right), m_{1} \cdot a\right)
$$

where $\psi^{12}: A \rightarrow \operatorname{Hom}_{k}\left(K_{1}, K_{2}\right)$ is a $k$-linear map. Since the action of $A$ must be associative, $\psi^{12}$ must be a derivation. In fact, it is a derivation that represents the extension $\tau_{2}$. 
Let $E_{3}$ be a right $A$-module that is an extension of $E_{2}$ by $K_{3}$, such that there is a short exact sequence $0 \rightarrow K_{3} \rightarrow E_{3} \rightarrow E_{2} \rightarrow 0$. Then $E_{3} \cong K_{3} \oplus E_{2} \cong K_{3} \oplus K_{2} \oplus K_{1}$ considered as a vector space over $k$, and the right action of $A$ is given by

$$
\left(m_{3}, m_{2}, m_{1}\right) \cdot a=\left(m_{3} \cdot a+\psi_{a}^{23}\left(m_{2}\right)+\psi_{a}^{13}\left(m_{1}\right), m_{2} \cdot a+\psi_{a}^{12}\left(m_{1}\right), m_{1} \cdot a\right)
$$

where $\psi^{i 3}: A \rightarrow \operatorname{Hom}_{k}\left(K_{i}, K_{3}\right)$ is a $k$-linear map for $i=1,2$. Since the action of $A$ must be associative, $\psi^{23}$ must be a derivation (representing the extension $\tau_{3}$ ), and $\psi^{13}$ must satsify

$$
-d\left(\psi^{13}\right)=\widetilde{\psi}_{13}, \quad \text { with } \widetilde{\psi}_{13}=\psi^{12} \cdot \psi^{23}
$$

such that the cup product $\tau_{2} \cup \tau_{3}=0$. It follows by an inductive argument that in the cofiltration

$$
E=E_{n} \stackrel{f_{n}}{\rightarrow} E_{n-1} \rightarrow \cdots \rightarrow E_{2} \stackrel{f_{2}}{\rightarrow} E_{1} \stackrel{f_{1}}{\rightarrow} E_{0}=0
$$

we have that $E=E_{n} \cong K_{n} \oplus \cdots \oplus K_{2} \oplus K_{1}$ considered as a vector space over $k$, with right action of $A$ given by

$$
\left(m_{n}, \ldots, m_{2}, m_{1}\right) \cdot a=\left(m_{n} \cdot a+\sum_{i=1}^{n-1} \psi_{a}^{i n}\left(m_{i}\right), \ldots, m_{2} \cdot a+\psi_{a}^{12}\left(m_{1}\right), m_{1} \cdot a\right)
$$

where $\psi^{i j}: A \rightarrow \operatorname{Hom}_{k}\left(K_{i}, K_{j}\right)$ is a 1 -cochain for $1 \leq i<j \leq n$. The condition that these cochains must satisfy for the action of $A$ to be associative, is that

$$
-d\left(\psi^{i j}\right)=\widetilde{\psi}_{i j}, \quad \text { with } \widetilde{\psi}_{i j}=\sum_{l=i+1}^{j-1} \psi^{i l} \cdot \psi^{l j}
$$

In other words, the family $\underline{\alpha}=\left\{\alpha_{i j}: 1 \leq i<j \leq n,(i, j) \neq(1, n)\right\}$ given by $\alpha_{i j}=$ $(-1)^{j-i+1} \psi^{i j}$ is a defining system for the matric Massey product

$$
\left\langle\tau_{2}, \tau_{3}, \ldots, \tau_{n}\right\rangle
$$

Moreover, the cohomology class of $\widetilde{\alpha}_{1 n}$ is zero, since $\widetilde{\alpha}_{1 n}=d\left(\alpha_{1 n}\right)$. This proves the following result:

Proposition 3 Let $n \geq 3$ be an integer, let $K_{1}, \ldots, K_{n}$ be simple A-modules in $\mathrm{S}$, and let $\tau_{2}, \ldots, \tau_{n}$ be extensions of $A$-modules with $\tau_{i} \in \operatorname{Ext}_{A}^{1}\left(K_{i-1}, K_{i}\right)$. There is an iterated extension in $\operatorname{Ext}(\mathrm{S})$ with $K_{1}, \ldots, K_{n}$ as its simple factors and with $\tau_{2}, \ldots, \tau_{n}$ as its simple extensions if and only if the matric Massey product $\left\langle\tau_{2}, \ldots, \tau_{n}\right\rangle$ is defined and contains zero.

\section{Iterated Extensions and Noncommutative Deformations}

Let $S=\left\{S_{\alpha}: \alpha \in I\right\}$ be a family of orthogonal points in an Abelian $k$-category $\mathcal{A}$, and let $\mathcal{A}(\mathrm{S})$ be the corresponding length category in $\mathcal{A}$. In this section, we consider the noncommutative deformations of finite subfamilies of $S$, and show that they determine the iterated extensions in $\operatorname{Ext}(S)$.

Let $(X, C)$ be an iterated extension in $\operatorname{Ext}(\mathrm{S})$ with order vector $\boldsymbol{\alpha}$. We define the extension type of $(X, C)$ to be the ordered quiver $\Gamma$ with nodes $\left\{\alpha_{1}, \alpha_{2}, \ldots, \alpha_{n}\right\}$ and edges $\gamma_{i-1, i}: \alpha_{i-1} \rightarrow \alpha_{i}$ for $2 \leq i \leq n$. This quiver is ordered in the sense that there is a total order $\gamma_{12}<\gamma_{23}<\cdots<\gamma_{n-1, n}$ on the edges in $\Gamma$. The extension type $\Gamma$ is determined by the order vector $\boldsymbol{\alpha}$, and isomorphic iterated extensions have the same extension type. We denote by $\mathcal{E}(S, \Gamma)$ the set of isomorphism classes of iterated extensions of the family $S$ with extension type $\Gamma$. 
Let $S(\Gamma) \subseteq \mathrm{S}$ be the set of simple factors of an iterated extension with extension type $\Gamma$. To fix notation, we shall write $S(\Gamma)=\left\{X_{1}, \ldots, X_{r}\right\}$. This means that for $1 \leq j \leq r$, we have that $X_{j}=S_{\alpha_{i}}$ for at least one value of $i$. Hence $r \leq n$, with $r<n$ if there are repeated factors.

We define the path algebra $k[\Gamma]$ of the ordered quiver $\Gamma$ to be the $k$-algebra with base consisting of paths $\gamma_{i-1, i} \cdot \gamma_{i, i+1} \cdots \gamma_{j-1, j}$ of length $j-i+1$ for $2 \leq i \leq j \leq n$, and with the following multiplicative strcuture: The product of two paths $\gamma \cdot \gamma^{\prime}$ is given by juxtaposition when the last arrow $\gamma_{j-1, j}$ in the first path $\gamma$ is the predecessor of the first arrow $\gamma_{j, j+1}$ in the second path $\gamma^{\prime}$ in the total ordering, and otherwise the product $\gamma \cdot \gamma^{\prime}=0$. We consider $e_{i}$ as a path of length 0 .

For example, an iterated extension of length $n=3$ with non-isomorphic simple factors $X_{1}=S_{\alpha_{1}}, X_{2}=S_{\alpha_{2}}$, and $X_{3}=S_{\alpha_{3}}$ has $r=3$, and its extension type $\Gamma$ has path algebra

$$
k[\Gamma]=\left(\begin{array}{ccc}
k e_{1} & k \gamma_{12} & k \gamma_{12} \gamma_{23} \\
0 & k e_{2} & k \gamma_{23} \\
0 & 0 & k e_{3}
\end{array}\right) \cong\left(\begin{array}{lll}
k & k & k \\
0 & k & k \\
0 & 0 & k
\end{array}\right)
$$

We remark that the path algebra $k[\Gamma]$ of any extension type $\Gamma$ is an object of the category $\mathrm{a}_{r}$ of Artinian $r$-pointed algebras. Recall that an Artinian $r$-pointed algebra is an Artinian $k$-algebra $R$ with $r$ simple modules fitting into a diagram $k^{r} \rightarrow R \rightarrow k^{r}$, where the composition is the identity.

Let us consider the noncommutative deformation functor Def $\mathrm{S}_{(\Gamma)}: \mathrm{a}_{r} \rightarrow$ Sets of the family $\mathrm{S}(\Gamma)=\left\{X_{1}, \ldots, X_{r}\right\}$, which is defined on the category $\mathrm{a}_{r}$. We refer to Eriksen et al. [7] for details of noncommutative deformations. We shall study the deformations in $\operatorname{Def}_{S(\Gamma)}(k[\Gamma])$. Without loss of generality, we may assume that the Abelian $k$-category $\mathcal{A}$ is the category of right modules over an associative $k$-algebra $A$. We remark that noncommutative deformations can be computed directly in many other Abelian $k$-categories as well, see for instance Eriksen [8] and Eriksen et al. [7].

Proposition 4 For any extension type $\Gamma$, there is a bijective correspondence between the noncommutative deformations in $\operatorname{Def}_{\mathrm{S}(\Gamma)}(k[\Gamma])$ and the set $\mathcal{E}(\mathrm{S}, \Gamma)$ of equivalence classes of iterated extensions of the family $\mathrm{S}$ with extension type $\Gamma$.

Proof Let $\boldsymbol{\alpha}$ be the order vector corresponding to the extension type $\Gamma$, and let $s$ be the unique index such that $S_{\alpha_{1}}=X_{s}$. Any noncommutative deformation $X_{\Gamma} \in \operatorname{Def}_{\mathrm{S}(\Gamma)}(k[\Gamma])$ has the form $X_{\Gamma}=\left(k[\Gamma]_{i j} \otimes_{k} X_{j}\right)$ as a left $k[\Gamma]$-module by flatness, with a right multiplication of $A$, and we consider the right $A$-submodule $X_{\Gamma}(s)=e_{s} \cdot X_{\Gamma} \subseteq X_{\Gamma}$. A path in $e_{s} \cdot k[\Gamma]$ is called leading if it has the form $\gamma_{12} \gamma_{23} \cdots \gamma_{i-1, i}$ and non-leading otherwise. By convention, we consider the path $e_{s}$ as leading, and define

$$
X_{\Gamma}^{N L}(s)=\bigoplus_{\gamma} \gamma \cdot X_{\Gamma} \subseteq X_{\Gamma}(s)
$$

where the sum is taken over all non-leading paths $\gamma$ in $e_{s} \cdot k[\Gamma]$. Notice that $X_{\Gamma}^{N L}(s) \subseteq$ $X_{\Gamma}(s)$ is closed under right multiplication by $A$. We define $X$ to be the right $A$-module $X=X_{\Gamma}(s) / X_{\Gamma}^{N L}(s)$. As a $k$-linear space, we have that

$$
X \cong \underset{1 \leq i \leq n}{\bigoplus}\left(\gamma_{12} \gamma_{23} \ldots \gamma_{i-1, i}\right) \otimes_{k} S_{\alpha_{i}}
$$


with $S_{\alpha_{i}}=X_{l}$ for some $l$ with $1 \leq l \leq r$, and we claim that there is a cofiltration $C$ of $X$ such that $(X, C)$ is an iterated extension of $S$ with extension type $\Gamma$. In fact, we may choose the cofiltration $C$ dual to the filtration $F$ given by

$$
F_{j}=\underset{j+1 \leq i \leq n}{\oplus}\left(\gamma_{12} \gamma_{23} \ldots \gamma_{i-1, i}\right) \otimes_{k} S_{\alpha_{i}}
$$

for $0 \leq j \leq n$, where $F_{j} \subseteq X$ is closed under right multiplication with $A$. Conversely, assume that an iterated extensions $(X, C)$ of $\mathrm{S}$ with extension type $\Gamma$ is given. Then it follows from Proposition 3 that the matric Massey product $\left\langle\tau_{2}, \tau_{3}, \ldots, \tau_{n}\right\rangle$ of its simple extensions is defined and contains zero. Hence, there is a defining system $\underline{\alpha}=\left\{\alpha_{i j}: 1 \leq\right.$ $i<j \leq n,(i, j) \neq(1, n)\}$ for this matric Massey product such that the cohomology class of $\widetilde{\alpha}_{1 n}$ is zero. By the construction preceding Proposition 3,X has the following form: As a $k$-linear vector space, $X \cong K_{n} \oplus K_{n-1} \oplus \cdots \oplus K_{2} \oplus K_{1}$ with $K_{i}=S_{\alpha_{i}}$, and the right multiplication of $A$ is given by

$$
\left(m_{n}, \ldots, m_{2}, m_{1}\right) \cdot a=\left(m_{n} \cdot a+\sum_{i=1}^{n-1} \psi_{a}^{i n}\left(m_{i}\right), \ldots, m_{2} \cdot a+\psi_{a}^{12}\left(m_{1}\right), m_{1} \cdot a\right)
$$

for $m_{i} \in K_{i}, a \in A$, where $\psi^{i j}=(-1)^{j-i+1} \alpha_{i j}$. This defines a right multiplication of $A$ on $X_{\Gamma}=\left(k[\Gamma]_{i j} \otimes_{k} X_{j}\right)$, and therefore a noncommutative deformation $X_{\Gamma}$ in $\operatorname{Def}_{S}(\Gamma)(k[\Gamma])$. In fact, the right multiplication is given by

$$
\left(e_{l} \otimes m_{l}\right) \cdot a=e_{l} \otimes\left(m_{l} \cdot a\right)+\sum_{i \in I_{l}} \sum_{i+1 \leq j \leq n}\left(\gamma_{i, i+1} \gamma_{i+1, i+2} \ldots \gamma_{j-1, j}\right) \otimes \psi_{a}^{i j}(m)
$$

for $1 \leq l \leq r, a \in A, m_{l} \in X_{l}$, with $I_{l}=\left\{i: \alpha_{i}=l\right\}$.

We say that $\mathrm{S}(\Gamma)$ is a swarm if $\operatorname{dim}_{k} \operatorname{Ext}_{A}^{1}\left(X_{i}, X_{j}\right)$ is finite for $1 \leq i, j \leq r$. We shall assume that this is the case in the rest of this section. In this case, the noncommutative deformation functor $\operatorname{Def}_{\mathrm{S}(\Gamma)}$ has a miniversal object $\left(H, X_{H}\right)$, consisting of a pro-representing hull $H$ of $\operatorname{Def}_{\mathrm{S}(\Gamma)}$ in the pro-category $\widehat{\mathrm{a}}_{r}$, and a versal family $X_{H} \in \operatorname{Def}_{\mathrm{S}(\Gamma)}(H)$; see Eriksen et al. [7] for details. We write $X(\mathrm{~S}, \Gamma)=\operatorname{Mor}(H, k[\Gamma])$ for the set of morphisms $\phi: H \rightarrow k[\Gamma]$ in $\widehat{\mathrm{a}}_{r}$. There is a natural map $X(\mathrm{~S}, \Gamma) \rightarrow \operatorname{Def}_{\mathrm{S}(\Gamma)}(k[\Gamma])$ given by $\phi \mapsto \operatorname{Def}_{\mathrm{S}(\Gamma)}(\phi)\left(X_{H}\right)$, and it is surjective by the versal property.

Lemma 5 The set $X(\mathrm{~S}, \Gamma)=\operatorname{Mor}(H, k[\Gamma])$ is an affine algebraic variety.

Proof Since $k[\Gamma]$ is an algebra in $\mathrm{a}_{r}$, and its radical $I(k[\Gamma])$ satisfy $I(k[\Gamma])^{n}=0$, any morphism $\phi: H \rightarrow k[\Gamma]$ in $\widehat{a}_{r}$ can be identified with $\phi_{n}: H_{n} \rightarrow k[\Gamma]$ since $\phi\left(I(H)^{n}\right)=0$. To prove that $X(\mathrm{~S}, \Gamma)=\operatorname{Mor}\left(H_{n}, k[\Gamma]\right)$ is an affine algebraic variety, it is enough to notice that $H_{n}$ is a quotient of $\mathrm{T}_{n}^{1}$, that $\operatorname{Mor}\left(\mathrm{T}_{n}^{1}, k[\Gamma]\right)$ is isomorphic to affine space $\mathbb{A}^{N}$, where

$$
N=\sum_{i, j} \operatorname{dim}_{k} \operatorname{Ext}_{A}^{1}\left(X_{i}, X_{j}\right) \cdot \operatorname{dim}_{k}\left(I(k[\Gamma]) / I(k[\Gamma])^{2}\right)_{i j}
$$

and that $\operatorname{Mor}\left(H_{n}, k[\Gamma]\right) \subseteq \operatorname{Mor}\left(\mathrm{T}_{n}^{1}, k[\Gamma]\right)$ is a closed subset in the Zariski topology, with equations given by the obstructions $f_{i j}(l)^{n} \in \mathrm{T}_{n}^{1}$ defining $H$.

Corollary 6 The set $\mathcal{E}(\mathrm{S}, \Gamma)$ of equivalence classes of iterated extensions of the family $\mathrm{S}$ with extension type $\Gamma$ is a quotient of the affine algebraic variety $X(\mathrm{~S}, \Gamma)$. 


\section{Uniserial Length Categories}

Let $\mathrm{S}$ be a family of orthogonal $k$-rational points in an Abelian $k$-category $\mathcal{A}$, and let $\mathcal{A}(\mathrm{S})$ be the corresponding length category. We say that an object $X$ in $\mathcal{A}(\mathrm{S})$ is uniserial if its lattice of subobjects is a chain. If this is the case, then this chain is the unique decomposition series of $X$. It follows that $X$ is uniserial if and only if any two cofiltrations of $X$ are isomorphic. Any uniserial object in $\mathcal{A}(\mathrm{S})$ is indecomposable, but the opposite implication does not hold in general. We say that $\mathcal{A}(\mathrm{S})$ is a uniserial category if every indecomposable object in $\mathcal{A}(\mathrm{S})$ is uniserial.

Theorem 7 (Gabriel) Let $\mathrm{S}=\left\{S_{\alpha}: \alpha \in I\right\}$ be a family of orthogonal $k$-rational points in an Abelian $k$-category $\mathcal{A}$. Then $\mathcal{A}(\mathrm{S})$ is uniserial if and only if the following conditions hold:

$$
\sum_{\beta \in I} \operatorname{dim}_{k} \operatorname{Ext}_{\mathcal{A}}^{1}\left(S_{\alpha}, S_{\beta}\right) \leq 1 \text { and } \sum_{\beta \in I} \operatorname{dim}_{k} \operatorname{Ext}_{\mathcal{A}}^{1}\left(S_{\beta}, S_{\alpha}\right) \leq 1 \quad \text { for all } \alpha \in \mathrm{S}
$$

Proof The result is due to Gabriel; see Gabriel [9] and Amdal, Ringdal [1]. A readable proof, due to $\mathrm{Yu} \mathrm{Ye}$, appears in Chen and Krause [3].

We denote by $\Lambda$ the Gabriel quiver of $\mathcal{A}(\mathrm{S})$, which has the objects in $S$ as nodes, indexed by $I$, and $\operatorname{dim}_{k} \operatorname{Ext}_{\mathcal{A}}^{1}\left(S_{\alpha}, S_{\beta}\right)$ arrows from node $\alpha$ to node $\beta$. Note that the condition in Theorem 7 is a condition on the Gabriel quiver. We shall assume that this condition is satisfied in the rest of this section, such that $\mathcal{A}(\mathrm{S})$ is a uniserial length category. Under this assumption, we shall classify and explicitly construct all indecomposable objects in $\mathcal{A}(\mathrm{S})$.

Let $(X, C)$ be an object of $\operatorname{Ext}(\mathrm{S})$ of length $n$ and with order vector $\boldsymbol{\alpha}$ such that $X$ is uniserial. Then it follows from Lemma 1.7.2 and the proof of Theorem 1.7.1 in Chen and Krause [3] that each $C_{i}$ in the cofiltration

$$
X=C_{n} \stackrel{f_{n}}{\rightarrow} C_{n-1} \rightarrow \cdots \rightarrow C_{2} \stackrel{f_{2}}{\rightarrow} C_{1} \stackrel{f_{1}}{\rightarrow} C_{0}=0
$$

is uniserial, and that the natural map $\operatorname{Ext}_{\mathcal{A}}^{1}\left(C_{i-1}, K_{i}\right) \rightarrow \operatorname{Ext}_{\mathcal{A}}^{1}\left(K_{i-1}, K_{i}\right)$ is an isomorphism for $2 \leq i \leq n$. This means in particular that $\tau_{i} \in \operatorname{Ext}_{\mathcal{A}}^{1}\left(K_{i-1}, K_{i}\right) \neq 0$ for $2 \leq i \leq n$. Hence the order vector $\boldsymbol{\alpha}$ corresponds to a path

$$
\alpha_{1} \rightarrow \alpha_{2} \rightarrow \cdots \rightarrow \alpha_{n}
$$

of length $n-1$ in the Gabriel quiver $\Lambda$. Furthermore, if $\left(X^{\prime}, C^{\prime}\right)$ is another object of $\operatorname{Ext}(\mathrm{S})$ with the same order vector $\alpha$ such that $X^{\prime}$ is uniserial, then $X \cong X^{\prime}$ in $\mathcal{A}$. This follows from Lemma 1.7.3 in Chen and Krause [3].

Let $\boldsymbol{\alpha} \in I^{n}$ be an order vector of length $n$. We say that $\boldsymbol{\alpha}$ is admissible if there is an iterated extension $(X, C)$ in $\operatorname{Ext}(\mathrm{S})$ with order vector $\alpha$ such that $X$ is uniserial. The comments above shows that if $\alpha$ is admissible, then it corresponds to a path of length $n-1$ in the Gabriel quiver $\Lambda$.

It also follows from the comments above that if $\alpha$ is an admissible order vector, then there is a unique uniserial object $X$ in $\mathcal{A}(\mathrm{S})$ with the property that it admits a cofiltration $C$ such that $(X, C)$ is an iterated extension in $\operatorname{Ext}(\mathrm{S})$ with order vector $\boldsymbol{\alpha}$. We shall write $X(\boldsymbol{\alpha})$ for this uniserial object.

Conversely, let $\alpha \in I^{n}$ be an order vector corresponding to a path of length $n-1$ in the Gabriel quiver $\Lambda$. If $n \geq 3$, then there are obstructions for $\alpha$ to be admissible. In fact, we have that $\operatorname{Ext}_{\mathcal{A}}^{1}\left(S_{\alpha_{i-1}}, S_{\alpha_{i}}\right) \neq 0$ for $2 \leq i \leq n$, and we may choose non-split extensions 
$\tau_{i} \in \operatorname{Ext}_{\mathcal{A}}^{1}\left(S_{\alpha_{i-1}}, S_{\alpha_{i}}\right)$, which are unique up to scalars in $k^{*}$. It follows from Proposition 3 that the order vector $\alpha$ is admissible if and only if the matric Massey product

$$
\left\langle\tau_{2}, \tau_{3}, \ldots, \tau_{n}\right\rangle \subseteq \operatorname{Ext}_{\mathcal{A}}^{2}\left(S_{\alpha_{1}}, S_{\alpha_{n}}\right)
$$

is defined an contains zero. The vanishing of these matric Massey products is clearly independent of the choice of non-split extensions $\tau_{i}$.

Notice that the condition of Proposition 3 can be interpreted as obstructions for lifting noncommutative deformations. In fact, let us write $\Gamma$ for the extension type corresponding to $\boldsymbol{\alpha}$, and let $I \subseteq k[\Gamma]$ be the Jacobson radical of the path algebra. Then the extensions $\tau_{2}, \ldots, \tau_{n}$ defines an infinitesimal noncommutative deformation in $\operatorname{Def}_{\mathrm{S}(\Gamma)}\left(k[\Gamma] / I^{2}\right)$, and $\boldsymbol{\alpha}$ is admissible if and only if this infinitesimal deformation can be lifted to the path algebra $k[\Gamma]$.

Theorem 8 Let $\mathrm{S}=\left\{S_{\alpha}: \alpha \in I\right\}$ be a family of orthogonal $k$-rational points in an Abelian $k$-category $\mathcal{A}$. If $\mathcal{A}(\mathrm{S})$ is a uniserial length category, then the indecomposable objects in $\mathcal{A}(\mathrm{S})$ of length $n$ are given by

$$
\left\{X(\boldsymbol{\alpha}): \boldsymbol{\alpha} \in I^{n} \text { is admissible }\right\}
$$

up to isomorphism in $\mathcal{A}(\mathrm{S})$. Moreover, an order vector $\boldsymbol{\alpha} \in I^{n}$ is admissible if and only if it satisfies the following conditions:

1. The order vector $\alpha$ corresponds to a path of length $n-1$ in the Gabriel quiver $\Lambda$.

2. When $n \geq 3$, the matric Massey product $\left\langle\tau_{2}, \tau_{3}, \ldots, \tau_{n}\right\rangle \subseteq \operatorname{Ext}_{\mathcal{A}}^{2}\left(S_{\alpha_{1}}, S_{\alpha_{n}}\right)$ is defined and contains zero for all non-split extensions $\tau_{i} \in \operatorname{Ext}_{\mathcal{A}}^{1}\left(S_{\alpha_{i-1}}, S_{\alpha_{i}}\right)$.

We note that if $\boldsymbol{\alpha}$ is admissible, then $X(\boldsymbol{\alpha})$ can be explicitly constructed from its simple factors and the simple extensions $\tau_{i} \in \operatorname{Ext}_{\mathcal{A}}^{1}\left(S_{\alpha_{i-1}}, S_{\alpha_{i}}\right)$. The construction is given by Proposition 3.

We say that $\mathcal{A}(\mathrm{S})$ is a hereditary length category if $\operatorname{Ext}_{\mathcal{A}}^{2}(S, T)=0$ for any objects $S, T$ in S. If this is the case, then the obstruction in Proposition 3 vanishes, and it follows that there is a bijective correspondence between paths of length $n-1$ in the Gabriel quiver $\Lambda$ and indecomposable objects in $\mathcal{A}(\mathrm{S})$.

\section{Graded Holonomic D-Modules on Monomial Curves}

Let $\Gamma \subseteq \mathbf{N}_{0}$ be a numerical semigroup, generated by positive integers $a_{1}, \ldots, a_{r}$ without common factors, and let $A=k[\Gamma] \cong k\left[t^{a_{1}}, \ldots, t^{a_{r}}\right]$ be its semigroup algebra over the field $k=\mathbb{C}$ of complex numbers. We call $A$ an affine monomial curve, and have that $X=\operatorname{Spec}(A)=\left\{\left(t^{a_{1}}, t^{a_{2}}, \ldots, t^{a_{r}}\right): t \in k\right\} \subseteq \mathbb{A}_{k}^{r}$.

We studied the positively graded algebra $D$ of differential operators on the monomial curve $A=k[\Gamma]$ in Eriksen [5], and the category grHol $_{D}$ of graded holonomic left $D$ modules in Eriksen [6]. We recall that any $D$-module $M$ satisfies the Bernstein inequality $d(M) \geq 1$, that $M$ is holonomic if $d(M)=1$, and that this condition holds if and only if $M$ has finite length; see Proposition 4 and Proposition 5 in Eriksen [6]. This implies that $\mathbf{g r H o l}_{D}$ is a length category, and its simple objects are given by

$$
\left\{M_{0}[w]: w \in \mathbf{Z}\right\} \cup\left\{M_{\alpha}[w]: \alpha \in J^{*}, w \in \mathbf{Z}\right\} \cup\left\{M_{\infty}[w]: w \in \mathbf{Z}\right\}
$$


where $J^{*}=\{\alpha \in \mathbb{C}: 0 \leq \operatorname{Re}(\alpha)<1, \alpha \neq 0\}$; see Theorem 10 in Eriksen [6]. Moreover, the graded extensions of the simple objects are given by

$$
\operatorname{Ext}_{D}^{1}\left(M_{\alpha}[w], M_{\beta}\left[w^{\prime}\right]\right)_{0}=\left\{\begin{array}{cc}
k \xi \cong k, & (\alpha, \beta)=(0, \infty),(\infty, 0) \text { and } w=w^{\prime} \\
k \xi \cong k, & \alpha=\beta \in J^{*} \text { and } w=w^{\prime} \\
0, & \text { otherwise }
\end{array}\right.
$$

for simple graded $D$-modules $M_{\alpha}[w], M_{\beta}\left[w^{\prime}\right]$ with $\alpha, \beta \in J^{*} \cup\{0, \infty\}$ and $w, w^{\prime} \in \mathbf{Z}$; see Proposition 12 in Eriksen [6].

Proposition 9 The family $\mathbf{S}=\left\{M_{\alpha}[w]: \alpha \in J^{*} \cup\{0, \infty\}, w \in \mathbf{Z}\right\}$ is the family of simple objects in $\mathbf{g r H o l}_{D}$, and it is a family of orthogonal $k$-rational points that satisfies the condition in Theorem 7. In particular, the category $\mathbf{g r H o l}_{D}$ of graded holonomic D-modules is a uniserial category.

Proof Since $k=\mathbb{C}$ is algebraically closed, it follows from the main theorem in Quillen [12] that $\operatorname{End}_{D}\left(M_{\alpha}[w]\right)=k$ for all $\alpha \in J^{*} \cup\{0, \infty\}, w \in \mathbf{Z}$. Moreover, the comments above show that $\mathrm{S}$ is the family of simple objects in $\mathbf{g r H o l}_{D}$, and therefore a family or orthogonal $k$-rational points, which satisfies the condition in Theorem 7.

It is, in principle, possible to construct all indecomposable objects in $\mathbf{g r H o l}_{D}$ using the constructive proof of Theorem 8 . As an illustration, we shall classify the indecomposable objects in the case $A=k[t]$, which is the unique smooth monomial curve. The classification would be similar in the other cases, since all rings of differential operators on monomial curves are Morita equivalent. However, the indecomposable objects would be defined by more complicated equations in the singular cases.

Note that when $A=k[t]$, the ring $D$ of differential operators on $A$ is the first Weyl algebra $A_{1}(k)=k[t]\langle\partial\rangle$, with generators $t$ and $\partial=d / d t$, and relation $[\partial, t]=1$. Let us write $E=t \partial$ for the Euler derivation in $D$. The simple objects in $\mathbf{g r H o l}_{D}$, up to graded isomorphisms and twists, are given by $M_{0}=D / D \partial, M_{\infty}=D / D t$ and $M_{\alpha}=D / D(E-\alpha)$ for $\alpha \in J^{*}$.

Theorem 10 Let $D=A_{1}(k)$ be the first Weyl algebra. The indecomposable graded holonomic D-module, up to graded isomorphisms and twists, are given by

$$
M(\alpha, n)=D / D(E-\alpha)^{n}, \quad M(\beta, n)=D / D \mathbf{w}(\beta, n)
$$

where $n \geq 1, \alpha \in J^{*}, \beta \in\{0, \infty\}$, and $\mathbf{w}(\beta, n)$ is the alternating word on $n$ letters in $t$ and $\partial$, ending with $\partial$ if $\beta=0$, and in $t$ if $\beta=\infty$.

Proof Let us write $I=J^{*} \cup\{0, \infty\}$, such that $\mathbf{S}=\left\{M_{\alpha}[w]:(\alpha, w) \in I \times \mathbf{Z}\right\}$ is the family of simple objects in $\mathbf{g r H o l}_{D}$. It follows from the computation of the graded extensios above that for any length $n \geq 1$ and any $(\alpha, w) \in I \times \mathbf{Z}$, there is a unique path

$$
(\alpha, w)=\left(\alpha_{1}, w_{1}\right) \rightarrow\left(\alpha_{2}, w_{2}\right) \rightarrow \cdots \rightarrow\left(\alpha_{n}, w_{n}\right)
$$

in $\Lambda$ such that $\operatorname{Ext}_{D}^{1}\left(M\left(\alpha_{i-1}\right)\left[w_{i-1}\right], M\left(\alpha_{i}\right)\left[w_{i}\right]\right)_{0} \neq 0$ for $2 \leq i \leq n$. The corresponding vector is admissible since $D=A_{1}(k)$ is a hereditary graded ring; see for instance Coutinho 
[4]. Note that if $\alpha \in J^{*}$, then $\alpha(i)=\alpha$ and $w_{i}=w$ for $1 \leq i \leq n$, and if $\alpha \in\{0, \infty\}$, then we have

$$
(\alpha(i), w(i))=\left\{\begin{array}{c}
(\alpha, w), \quad i \text { is odd } \\
(0, w-1), \quad i \text { is even, } \alpha=\infty \\
(\infty, w+1), \quad i \text { is even, } \alpha=0
\end{array}\right.
$$

for $1 \leq i \leq n$. The rest follows from Theorem 8 and the following comments, and the fact that graded extensions obtained using factorization in $D$, such as

$$
0 \rightarrow D / D(E-\alpha) \rightarrow D /(E-\alpha)^{n} \rightarrow D / D(E-\alpha)^{n-1} \rightarrow 0
$$

for $\alpha \in J^{*}$ and $n \geq 2$, are non-split.

Let $\mathcal{O}$ be the ring of germs of holomorphic functions around 0 in $X=\mathbb{C}$, and let $\mathcal{D}$ be the ring of differential operators on $\mathcal{O}$. The classification of the indecomposable regular holonomic $\mathcal{D}$-modules is due to Boutet de Monvel [2], given by

$$
\mathcal{D} /(z D-\alpha)^{n}(\alpha \notin \mathbb{Z}), \quad \mathcal{D} /(\ldots z D z D), \quad \mathcal{D} /(\ldots D z D z)
$$

We note that this classical result correspond well with our classification of graded holonomic $D$-modules in Theorem 10 .

\section{A Non-Hereditary Example}

Let $k$ be an algebraically closed field, and let $A=k[x] /\left(x^{2}\right)$. There is a unique simple $A$ module $S=A /(x)$, and the family $S=\{S\}$ is clearly a family of orthogonal $k$-rational points in the category $\mathcal{A}$ of left $A$-modules. Moreover, a simple computation shows that

$$
\operatorname{Ext}_{A}^{1}(S, S)=k \cdot \xi \cong k, \quad \operatorname{Ext}_{A}^{2}(S, S)=k \cdot \kappa \cong k
$$

hence $\mathcal{A}(\mathrm{S})$, which we identify with the category of left $A$-modules of finite length, is a uniserial length category that is not hereditary. There is an indecomposable module of length $n=2$, given by the non-split extension

$$
0 \rightarrow(x) /\left(x^{2}\right) \rightarrow k[x] /\left(x^{2}\right) \rightarrow k[x] /(x) \rightarrow 0
$$

This is of course the regular representation $M=A$. However, another simple computation shows that the cup product (second order matric Massey product) $\langle\xi, \xi\rangle=\kappa \neq 0$. This means that there is no indecomposable module with order vector $\boldsymbol{\alpha}$ when $\boldsymbol{\alpha}$ corresponds to a path in the Gabriel quiver of length $n-1 \geq 2$. We conclude that the simple module $S=A /(x)$ and regular module $A$ is a complete list of indecomposable left $A$-modules in $\mathcal{A}(\mathrm{S})$.

Funding Information Open Access funding provided by Norwegian Business School.

Open Access This article is licensed under a Creative Commons Attribution 4.0 International License, which permits use, sharing, adaptation, distribution and reproduction in any medium or format, as long as you give appropriate credit to the original author(s) and the source, provide a link to the Creative Commons licence, and indicate if changes were made. The images or other third party material in this article are included in the article's Creative Commons licence, unless indicated otherwise in a credit line to the material. If material is not included in the article's Creative Commons licence and your intended use is not permitted by statutory regulation or exceeds the permitted use, you will need to obtain permission directly from the copyright holder. To view a copy of this licence, visit http://creativecommonshorg/licenses/by/4.0/. 


\section{References}

1. Amdal, I.K., Ringdal, F.: Catégories unisérielles. C. R. Acad. Sci. Paris Sér A-B 267, A247-A249 (1968)

2. Boutet de Monvel, L.: D-modules holonômes réguliers en une variable. Mathematics and Physics (Paris, 1979/1982), Progr. Math., vol. 37, pp. 313-321. Birkhäuser, Boston (1983). MR 728427

3. Chen, X.-W., Krause, H.: Introduction to coherent sheaves on weighted projective lines. arXiv:0911.4473 (2009)

4. Coutinho, S.C.: A Primer of Algebraic D-Modules London Mathematical Society Student Texts, vol. 33. Cambridge University Press, Cambridge (1995)

5. Eriksen, E.: Differential operators on monomial curves. J. Algebra 264(1), 186-198 (2003)

6. Eriksen, E.: Graded Holonomic D-modules on Monomial Curves. arXiv:1803.04367 (2018)

7. Eriksen, E., Laudal, O.A., Siqveland, A.: Noncommutative Deformation Theory, Monographs and Research Notes in Mathematics. CRC Press, Boca Raton (2017)

8. Eriksen, E.: Computing noncommutative deformations of presheaves and sheaves of modules. Canad. J. Math. 62(3), 520-542 (2010). MR 2666387

9. Gabriel, P.: Indecomposable Representations. II, Symposia Mathematica, Vol. XI (Convegno di Algebra Commutativa, INDAM, Rome, 1971), pp. 81-104. Academic Press, London (1973)

10. Laudal, O.A.: Noncommutative deformations of modules. Homology Homotopy Appl. 4(2), part 2, 357396 (2002). The Roos Festschrift volume, 2

11. May, J.P.: Matric Massey products. J. Algebra 12, 533-568 (1969). MR 238929

12. Quillen, D.: On the endomorphism ring of a simple module over an enveloping algebra. Proc. Amer. Math. Soc. 21, 171-172 (1969)

13. Ringel, C.M.: Representations of $K$-species and bimodules. J. Algebra 41(2), 269-302 (1976)

Publisher's Note Springer Nature remains neutral with regard to jurisdictional claims in published maps and institutional affiliations. 